\title{
NONOSCILLATORY SOLUTIONS \\ OF SECOND ORDER DIFFERENTIAL EQUATIONS WITH INTEGRABLE COEFFICIENTS
}

\author{
MANABU NAITO \\ (Communicated by Kenneth R. Meyer)
}

\begin{abstract}
The asymptotic behavior of nonoscillatory solutions of the equation $x^{\prime \prime}+a(t)|x|^{\gamma} \operatorname{sgn} x=0, \gamma>0$, is discussed under the condition that $A(t)=$ $\lim _{T \rightarrow \infty} \int_{t}^{T} a(s) d s$ exists and $A(t) \geq 0$ for all $t$. For the sublinear case of $0<\gamma<1$, the existence of at least one nonoscillatory solution is completely characterized.
\end{abstract}

\section{INTRODUCTION}

Consider the second-order differential equation

$$
x^{\prime \prime}+a(t)|x|^{\gamma} \operatorname{sgn} x=0,
$$

where $a$ is continuous on $\left[t_{0}, \infty\right)$ and $\gamma$ is a positive constant. Equation (1.1) is called the generalized Emden-Fowler equation, and is classified as superlinear or sublinear according to $\gamma>1$ or $0<\gamma<1$. If $\gamma=1$, then (1.1) reduces to a familiar linear equation.

In this paper we study the asymptotic properties of nonoscillatory solutions of equation (1.1) under the condition that

$$
\lim _{t \rightarrow \infty} \int_{t_{0}}^{t} a(s) d s \quad \text { exists and is finite. }
$$

If condition (1.2) is satisfied, then we can introduce the function $A$ by

$$
A(t)=\int_{t}^{\infty} a(s) d s, \quad t \geq t_{0} .
$$

Our purpose here is to extend the well-known results for the case where $a(t)$ is nonnegative to the more general case where $A(t)$ is nonnegative. In the case where $A(t)$ is nonnegative, we can obtain a necessary and sufficient oscillation criterion for the sublinear equation.

Received by the editors August 20, 1989 and, in revised form, October 16, 1989.

1980 Mathematics Subject Classification (1985 Revision). Primary 34C10, 34C11. 
If $a(t) \geq 0$ for $t \geq t_{0}$, then it is easily seen that a nonoscillatory solution $x$ of (1.1) satisfies exactly one of the following three asymptotic conditions:

$$
\begin{aligned}
& x(t)=c+o(1) \quad \text { as } t \rightarrow \infty, \quad \text { where } c \neq 0 ; \\
& x(t)=o(t) \quad \text { and } \quad \lim x(t)= \pm \infty \quad \text { as } t \rightarrow \infty ; \\
& x(t)=c t+o(t) \quad \text { as } t \rightarrow \infty, \quad \text { where } c \neq 0 .
\end{aligned}
$$

We can prove that this fact remains valid even for the case where $A(t) \geq 0$ for $t \geq t_{0}$ :

Theorem 1.1. Let $\gamma>0$. Suppose that (1.2) holds and that $A(t) \geq 0$ for $t \geq t_{0}$. Then, for each nonoscillatory solution $x$ of (1.1), exactly one of the three asymptotic conditions (1.4)-(1.6) is satisfied.

For the superlinear case, the next theorem is already known.

Theorem 1.2. Let $\gamma>1$. Suppose that (1.2) holds and that $A(t) \geq 0$ for $t \geq t_{0}$. Then the following three statements are equivalent:

(i) equation (1.1) has a nonoscillatory solution $x$ satisfying (1.4);

(ii) equation (1.1) has a nonoscillatory solution;

(iii) the two integral conditions below are satisfied:

$$
\int^{\infty} A(t) d t<\infty \text { and } \int^{\infty} t A^{2}(t) d t<\infty .
$$

In fact, the equivalence of (ii) and (iii) can be obtained as the contrapositive form of the result of Butler [1, Theorem 2.3]. Note that Butler's result is applicable to the more general case. The equivalence of (i) and (iii) has been proved by Naito [6, Theorem 2.4]. In light of the "duality" between superlinear and sublinear equations for the case of $a(t) \geq 0$ (see Coffman and Wong [3]) and the result of Naito [6, Theorem 3.5], it is natural to conjecture that the next theorem is true.

Theorem 1.3. Let $0<\gamma<1$. Suppose that (1.2) holds and that $A(t) \geq 0$ for $t \geq t_{0}$. Then the following three statements are equivalent:

(i) equation (1.1) has a nonoscillatory solution $x$ satisfying (1.6);

(ii) equation (1.1) has a nonoscillatory solution;

(iii) the two integral conditions below are satisfied:

$$
\int^{\infty} t^{\gamma-1} A(t) d t<\infty \text { and } \int^{\infty} t^{2 \gamma-1} A^{2}(t) d t<\infty .
$$

In the succeeding section we show that Theorems 1.1 and 1.3 are indeed true. It is easily seen that the equivalence of (ii) and (iii) in Theorem 1.3 can be restated as follows:

Corollary 1.4. Let $0<\gamma<1$. Suppose that (1.2) holds and that $A(t) \geq 0$ for $t \geq t_{0}$. Then all solutions of (1.1) are oscillatory if and only if

$$
\int^{\infty}\left(t^{\gamma-1} A(t)+\int_{t}^{\infty}\left[s^{\gamma-1} A(s)\right]^{2} d s\right) d t=\infty .
$$


The problem of oscillation of solutions to the Emden-Fowler equation (1.1) and the more general equation $x^{\prime \prime}+a(t) f(x)=0$ has attracted a great deal of attention, and numerous results have been obtained. For a general discussion on this problem, we refer to the survey article of Wong [10] (the nonlinear case) and the book of Swanson [7] (the linear case). The case where (1.2) holds has been studied by several authors including Butler [1, 2], Coles [4], Kwong and Wong [5], Naito [6], Willett [8], and Wong [9].

\section{Proofs of Theorems 1.1 AND 1.3}

Proof of Theorem 1.1. Let $x$ be a nonoscillatory solution of (1.1). There is no loss of generality in supposing that $t_{0}>0$ and $x(t)>0$ for $t \geq t_{0}$. It is known (Kwong and Wong [5, Theorem 1]) that $x(t)$ satisfies the equality

$$
x^{\prime}(t)=A(t) x^{\gamma}(t)+\gamma x^{\gamma}(t) \int_{t}^{\infty} x^{-\gamma-1}(s) x^{\prime 2}(s) d s
$$

for $t \geq t_{0}$, where $A$ is defined by (1.3). Therefore we have

$$
x^{\prime}(t) \geq A(t) x^{\gamma}(t), \quad t \geq t_{0} .
$$

Further, by the nonnegativity of $A(t)$ we have $x^{\prime}(t) \geq 0$ for $t \geq t_{0}$. An integration by parts of (1.1) gives

$$
x^{\prime}(\tau)-A(\tau) x^{\gamma}(\tau)+\gamma \int_{t}^{\tau} A(s) x^{\gamma-1}(s) x^{\prime}(s) d s=x^{\prime}(t)-A(t) x^{\gamma}(t)
$$

for $\tau \geq t \quad\left(\geq t_{0}\right)$. Let $t$ be fixed. Since $A(s) x^{\gamma-1}(s) x^{\prime}(s)$ is nonnegative, the integral term in (2.2) has a finite limit or diverges to $\infty$ as $\tau \rightarrow \infty$. If the latter case occurs, then $x^{\prime}(\tau)-A(\tau) x^{\gamma}(\tau) \rightarrow-\infty$ as $\tau \rightarrow \infty$, which is a contradiction to $(2.1)$. Thus the former case occurs:

$$
\int^{\infty} A(s) x^{\gamma-1}(s) x^{\prime}(s) d s<\infty .
$$

This implies that the function $K_{1}$ can be defined by

$$
K_{1}(t)=\int_{t}^{\infty} A(s) x^{\gamma-1}(s) x^{\prime}(s) d s, \quad t \geq t_{0},
$$

and that $x^{\prime}(\tau)-A(\tau) x^{\gamma}(\tau)$ converges to a finite limit as $\tau \rightarrow \infty$. Let $\alpha$ be the limit:

$$
\alpha=\lim _{\tau \rightarrow \infty}\left[x^{\prime}(\tau)-A(\tau) x^{\gamma}(\tau)\right] .
$$

Then equality (2.2) yields

$$
x^{\prime}(t)=\alpha+A(t) x^{\gamma}(t)+\gamma K_{1}(t)
$$

for $t \geq t_{0}$. Observe by (2.1) that $\alpha \geq 0$. From (2.1) and (2.3) it follows that

$$
\int^{\infty} A^{2}(s) x^{2 \gamma-1}(s) d s<\infty .
$$


Therefore the function $K_{2}$ can be defined by

$$
K_{2}(t)=\int_{t}^{\infty} A^{2}(s) x^{2 \gamma-1}(s) d s, \quad t \geq t_{0} .
$$

Integrating (2.5) over $\left[t_{0}, t\right]$, we have

$$
x(t)=x\left(t_{0}\right)+\alpha\left(t-t_{0}\right)+\int_{t_{0}}^{t} A(s) x^{\gamma}(s) d s+\gamma \int_{t_{0}}^{t} K_{1}(s) d s
$$

for $t \geq t_{0}$. By Schwarz's inequality and the fact that $x^{\prime}(t) \geq 0$ for $t \geq t_{0}$, the first integral term in (2.8) can be estimated as follows:

$$
\begin{aligned}
& \int_{t_{0}}^{t} A(s) x^{\gamma}(s) d s \\
& \quad \leq\left(\int_{t_{0}}^{t} A^{2}(s) x^{2 \gamma-1}(s) d s\right)^{1 / 2}\left(\int_{t_{0}}^{t} x(s) d s\right)^{1 / 2} \\
& \quad \leq K_{2}^{1 / 2}\left(t_{0}\right)\left(t-t_{0}\right)^{1 / 2} x^{1 / 2}(t), \quad t \geq t_{0} .
\end{aligned}
$$

Thus we obtain

$$
x(t) \leq x\left(t_{0}\right)+\alpha\left(t-t_{0}\right)+K_{2}^{1 / 2}\left(t_{0}\right)\left(t-t_{0}\right)^{1 / 2} x^{1 / 2}(t)+\gamma K_{1}\left(t_{0}\right)\left(t-t_{0}\right)
$$

for $t \geq t_{0}$. The above inequality may be regarded as a quadratic inequality with respect to $x^{1 / 2}(t)$. Then we find that

$$
x^{1 / 2}(t) \leq\left[K_{2}^{1 / 2}\left(t_{0}\right)\left(t-t_{0}\right)^{1 / 2}+D^{1 / 2}(t)\right] / 2, \quad t \geq t_{0},
$$

where

$$
D(t)=K_{2}\left(t_{0}\right)\left(t-t_{0}\right)+4\left[x\left(t_{0}\right)+\alpha\left(t-t_{0}\right)+\gamma K_{1}\left(t_{0}\right)\left(t-t_{0}\right)\right], \quad t \geq t_{0} .
$$

It is obvious that $D(t)=O(t)$ as $t \rightarrow \infty$, and consequently, there exists a positive constant $M$ such that

$$
x(t) \leq M t \quad \text { for } t \geq t_{0} .
$$

Let $T\left(\geq t_{0}\right)$ be an arbitrary number. It is clear that

$$
0 \leq \frac{1}{t} \int_{t_{0}}^{t} A(s) x^{\gamma}(s) d s=\frac{1}{t} \int_{t_{0}}^{T} A(s) x^{\gamma}(s) d s+\frac{1}{t} \int_{T}^{t} A(s) x^{\gamma}(s) d s
$$

for $t \geq T$. Arguing as in (2.9), we have

$$
\int_{T}^{t} A(s) x^{\gamma}(s) d s \leq K_{2}^{1 / 2}(T)(t-T)^{1 / 2} x^{1 / 2}(t), \quad t \geq T,
$$

which, combined with (2.10), yields

$$
\int_{T}^{t} A(s) x^{\gamma}(s) d s \leq M^{1 / 2} K_{2}^{1 / 2}(T) t^{1 / 2}(t-T)^{1 / 2}, \quad t \geq T .
$$


Taking the upper limit as $t \rightarrow \infty$ in (2.11) and using (2.12), we see that

$$
0 \leq \limsup _{t \rightarrow \infty} \frac{1}{t} \int_{t_{0}}^{t} A(s) x^{\gamma}(s) d s \leq M^{1 / 2} K_{2}^{1 / 2}(T) .
$$

Since $T$ is arbitrary and $K_{2}(T)$ tends to zero as $T \rightarrow \infty$, letting $T \rightarrow \infty$ in (2.13), we find that

$$
\lim _{t \rightarrow \infty} \frac{1}{t} \int_{t_{0}}^{t} A(s) x^{\gamma}(s) d s=0 .
$$

In view of (2.8), (2.14), and the fact that $K_{1}(t) \rightarrow 0$ as $t \rightarrow \infty$, we get

$$
\lim _{t \rightarrow \infty} x(t) / t=\alpha \text {. }
$$

Recall that $x(t)$ is nondecreasing for $t \geq t_{0}$. Then there are three possibilities: (i) $\alpha=0$ and $x(t)$ is bounded above; (ii) $\alpha=0$ and $x(t)$ is unbounded; (iii) $\alpha>0$ (and hence $x(t)$ is unbounded). Case (i) implies (1.4) with $c=\lim _{t \rightarrow \infty} x(t)>0$, while case (iii) implies (1.6) with $c=\alpha>0$. It is also clear that case (ii) implies (1.5). The proof of Theorem 1.1 is complete.

Proof of Theorem 1.3. It is trivial that (i) implies (ii). The equivalence of (i) and (iii) has been proved in [6, Theorem 3.5]. Here we claim that (ii) implies (iii). Butler [2] has showed that if

$$
\int^{\infty} t^{\gamma-1} A(t) d t=\infty
$$

then all solutions of (1.1) are oscillatory. This means that if (1.1) has a nonoscillatory solution, then the first integral condition in (1.8) is satisfied. Therefore it is enough to show that if $(1.1)$ has a nonoscillatory solution, then the second integral condition in (1.8) is satisfied:

$$
\int^{\infty} t^{2 \gamma-1} A^{2}(t) d t<\infty
$$

Suppose that equation (1.1) has a solution $x(t)$ which is positive on $\left[t_{0}, \infty\right)$. The equalities and the inequalities in the proof of Theorem 1.1 remain valid. Consider the case where $2 \gamma-1 \leq 0$, then the desired condition (2.15) follows from (2.6) and (2.10). Next consider the case where $2 \gamma-1>0$. In (2.8), the first three terms of the right-hand side are nonnegative, and $K_{1}(t)$ is nonincreasing on $\left[t_{0}, \infty\right)$. Thus we have

$$
x(t) \geq \gamma\left(t-t_{0}\right) K_{1}(t), \quad t \geq t_{0} .
$$

Hence, in view of (2.1), (2.4), and (2.7), we get

$$
x(t) \geq \gamma\left(t-t_{0}\right) K_{2}(t), \quad t \geq t_{0},
$$

and so, by the assumption $2 \gamma-1>0$,

$$
A^{2}(t) x^{2 \gamma-1}(t) K_{2}^{-2 \gamma+1}(t) \geq \gamma^{2 \gamma-1}\left(t-t_{0}\right)^{2 \gamma-1} A^{2}(t), \quad t \geq t_{0} .
$$


Since $K_{2}^{\prime}(t)=-A^{2}(t) x^{2 \gamma-1}(t)$ for $t \geq t_{0}$, an integration of (2.16) gives

$$
-\frac{1}{2-2 \gamma} K_{2}^{-2 \gamma+2}(t)+\frac{1}{2-2 \gamma} K_{2}^{-2 \gamma+2}\left(t_{0}\right) \geq \gamma^{2 \gamma-1} \int_{t_{0}}^{t}\left(s-t_{0}\right)^{2 \gamma-1} A^{2}(s) d s
$$

for $t \geq t_{0}$. Note that the left-hand side of the above inequality is bounded on $\left[t_{0}, \infty\right)$. Then it is easy to see that the desired condition (2.15) is also satisfied. The proof of Theorem 1.3 is complete.

\section{REFERENCES}

1. G. J. Butler, On the oscillatory behaviour of a second order nonlinear differential equation, Ann. Mat. Pura Appl. 105 (1975), 73-92.

2. __ An integral criterion for the oscillation of a second order sublinear ordinary differential equation, Indian J. Math. 24 (1982), 1-7.

3. C. V. Coffman and J. S. W. Wong, Oscillation and nonoscillation theorems for second order ordinary differential equations, Funkcial. Ekvac. 15 (1972), 119-130.

4. W. J. Coles, Oscillation criteria for nonlinear second order equations, Ann. Mat. Pura Appl. 82 (1969), 123-133.

5. M. K. Kwong and J. S. W. Wong, An application of integral inequality to second order nonlinear oscillation, J. Differential Equations 46 (1982), 63-77.

6. M. Naito, Asymptotic behavior of solutions of second order differential equations with integrable coefficients, Trans. Amer. Math. Soc. 282 (1984), 577-588.

7. C. A. Swanson, Comparison and oscillation theory of linear differential equations, Academic Press, New York, 1968.

8. D. Willett, On the oscillatory behavior of the solutions of second order linear differential equations, Ann. Polon. Math. 21 (1969), 175-194.

9. J. S. W. Wong, Oscillation and nonoscillation of solutions of second order linear differential equations with integrable coefficients, Trans. Amer. Math. Soc. 144 (1969), 197-215.

10. __, On the generalized Emden-Fowler equation, SIAM Rev. 17 (1975), 339-360.

Department of Mathematics, Faculty of Science, Hiroshima University, Hiroshima 730, JAPAN 